\title{
The modern use of optics design techniques to production visual Art works
}

\author{
Diaa Ahmed Mohamed Ahmedien \\ Assistant lecture Department of Drawing and painting, Faculty of art education, University of elwan, Egypt.
}

\begin{abstract}
In this work, I did my best to reveal the possibility of using laser light to produce the visual art generally and as visual language in particular. Many optical designs have been built by using and controlling the path of laser beam to achieve the target of our work. The main objective of this work is to study the effect of the optical design variables in the formation methods of laser beam to produce visual art. From here we can say that, this research is considered an extension of the close relationship between optics science and art throughout the ages. In addition to these results of this research have become visual language in itself, as a result of using the close relationship between optics science and art in produced it.
\end{abstract}

\section{Introduction}

A good relationship has been created particularly between art and science of optics for a long time, because it looks at the visual field which is the main topic in the visual art today. Since the invention of Ibn alHaitham to the dark room then Leonardo Da Vinci came after him and recorded his observations upon, and put the lens on the hole of room to control in the size of the reflected image [1].

The science of optics had been called natural magic in the seventeenth century and earlier [2]. The natural magic terms varied after that, therefor they have been expanded and placed inside the other taxonomic groups such as but not limited the entertainment arts, technology, and natural science. It has been one of the main reasons behind continuing the natural magic this visual scientific side that distinguished it [3]. This term has been developed even has become science in itself, called geometrical optics [4]. First it specialized in studying the impact of lenses and Mirrors in influence the vision, the path of light and its behavior. Geometrical optics has varied applications in various fields through wide evolutionary mutations, it have been started by invented photography machine (the Camera) in 1839 as a static image [4], and evolved "where it stood behind one of the most important inventions in the late nineteenth and early twentieth century, it is the cinema [5].

Then optics has been evolved through enter the modern applications and uses of the laser [6]. Since the laser has been discovered, the scientists and specialists in their respective fields are interested in applications that can be available in many areas through the use of laser technology, but it is clear that the majority of applied research that dealt with the laser was distantly related for visual art as a visual language.

The researcher, as an artist, denies the conception that technology leads to the alienation of human from nature, or rather the modern day person from his natural self. Therefore, if there lays a problem in the human interpretation of technology it is due to the error in application or use but not in the technology per se for it is nothing but a tool that manifests the condensation of human understanding of the natural laws. The question becomes how could the laws that carry out the essence of nature contradict with nature?!!!, The truth is that there is no contradiction whatsoever, and that the common ground between technology and nature is subtle which we consider signify more value on harmony between the two, as an example, when we consider a laser beam as a technology we see light as in nature but in a more intense way, since the laser is no ordinary light, but is a product of applying certain conditions on the way it is produced. The utilization of any optical component like a lens, a mirror, or a prism replicates what happens in nature, for example, the interference of light through petrochemicals (oil) and water produces a rainbow as did Newton when he used a glass prism to disperse the white light.

From here this paper is trying to answer the following two questions:

1 - To what extent can the use of optics science with the laser beam to obtain the different visual effects formulas and techniques?

2 - How can the visual arts to benefit from visual optical formulas to produce visual designs?

\section{Material and Methodology}

The first characteristic of a laser beam is to intensify and coherence its beam. With note that there are two kinds of coherence in laser beam temporal coherence and spatial coherence ${ }^{[7]}$, where we depended on these coherence property kinds of laser beam, which cannot be ignored when we are in the process of establishing the visual form by using a laser beam at this experiment. The Nd: YAG laser has been used in this experiment. 
We tested the possibility of measurements of transverse radiation mode, and were able to confirm the properties of the green (Nd: YAG) laser beam, which it used in the experiment through the software of the laser beam profile analysis. Figure 1 shows the Optical setup for laser beam profile analysis, where the laser beam has high energy, so we must split the energy of beam by splitter (50-50), to reduce of the beam energy to fit with the sensitivity of camera ${ }^{[8]}$, then a beam passes from three filters, which have rate of attenuation $\left(10^{-7}\right)$ to fit the Sensitivity of camera ${ }^{[9]}$. The beam profile has been recorded on the electronic laser beam analysis device (spiricon: LBA-100A), through the computer screen.

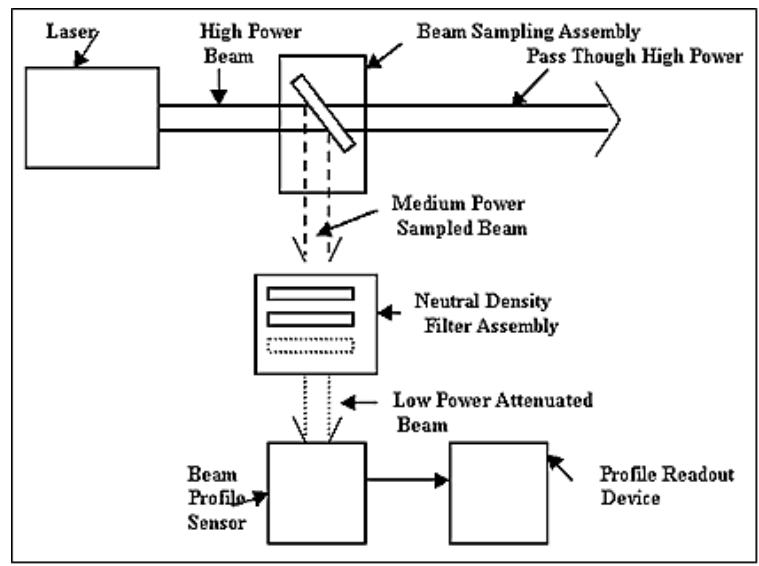

Fig.1: Optical setup for electronic laser beam analysis ${ }^{[10]}$.

The significance of the beam profile is the energy density, the concentration, and the collimation of the light which are all affected by it ${ }^{[11]}$. Also the propagation of the beam through space is significantly affected on the beam profile ${ }^{[12]}$. We calculated $\mathrm{M}^{2}$, or the factor $\mathrm{k}=1 / \mathrm{M}^{2}{ }^{[13]}$, which is important in describing the quality of laser beam in many applications, especially those in which a Gaussian beam is the desired profile. Figure 2 shows the position of the detector from the lens, where it has been placed in the focus of lens at distance $5 \mathrm{~cm}$.

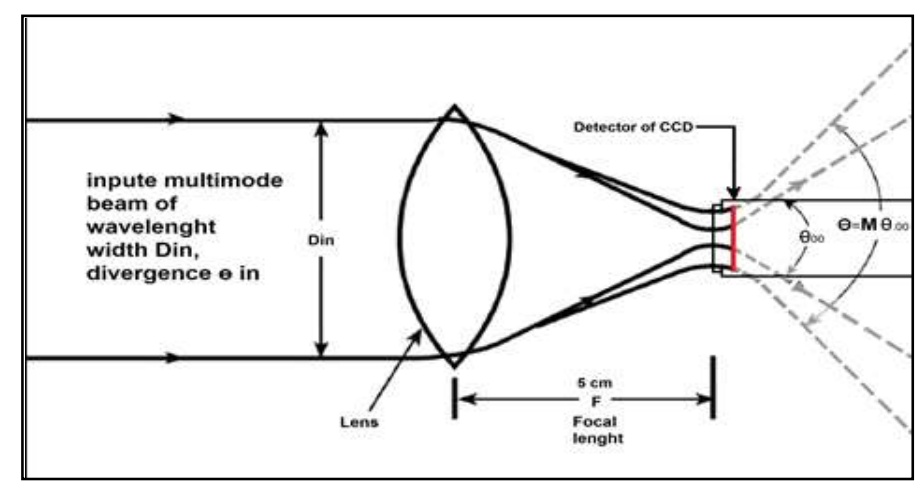

Fig.2: Curve showing $\mathrm{M}^{2}$ : Characteristics and Detector position of the lens [14].

Figure 3-A shows the waist of the laser beam profile at a focal length of lens and figure 3-B shows the laser beam profile when the laser beam was beyond the Rayleigh range

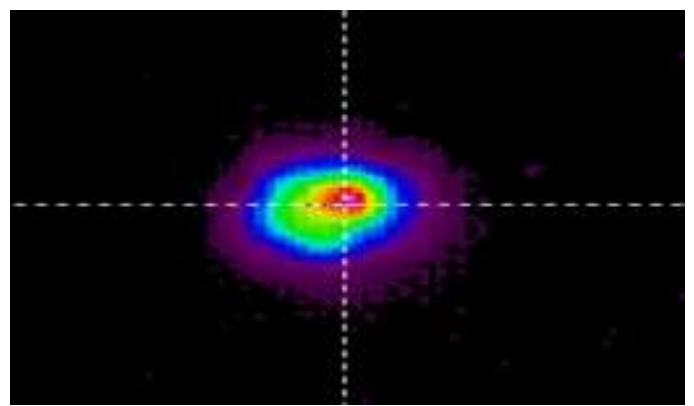

Fig3-A: Beam profile of CW Nd:YAG Laser ${ }^{[15]}$ Wavelength $=532 \mathrm{~nm}, 4 \mathrm{X}$ Beam diameter $\left(\mathrm{W}_{0}\right)(\mathrm{W} 0): 113.195$

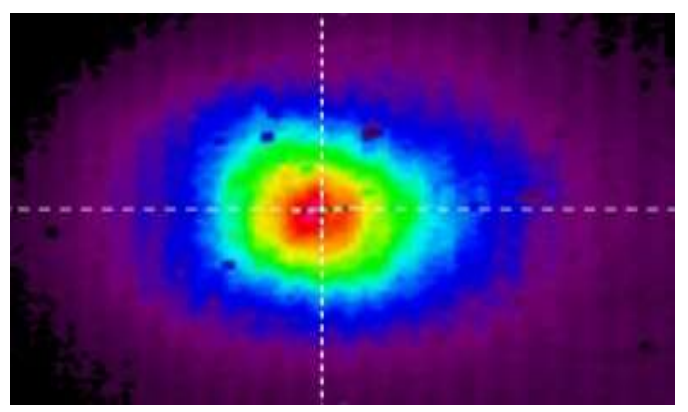

Fig3-B: Beam profile of CW Nd:YAG Laser ${ }^{[15]}$ Wavelength $=532$ nm, Diameter: $1522.34\left(\mathrm{~W}_{1}\right): 761.17$ 
Know that $\mathrm{M}^{2}$ calculator equation is: $\quad \mathrm{M}^{2}={\frac{\pi \mathrm{W}_{\mathrm{I}} \mathrm{W}_{0}}{4 \lambda \mathrm{F}}}^{{ }^{[16]}}$

By Compensation in the equation, it is clear that $\mathrm{M}^{2}=2.5$.

Through the above it is clear, the beam of laser which has been used in this Experiment, multi-modes.

\section{Experimental Set up}

We depended on interference concept of optics to build the setups of the optical designs. This concept makes us think about these designs and all its variables, depending on the principle which have been built upon. Therefore we have conducted several experiments, to test the possibility of this concept in forming the beam of green (Nd: YAG) laser, using a set of complex optical designs as follows:

First Optical Design-(Depending on Michelson Interferometer)

This design is the basis for the rest of the designs used in this experiment and has been built depending on Michelson interferometer fundamentals ${ }^{[17,18]}$.

In this design the laser source has been put on the left side, to incident on a splitter (1), it has percentage of permeability up to $10 \%$. Therefore the path of beam has been split into two paths; the first path is up to the top, where incident on mirror (2) to reflected down again, the other path Passes through the splitter (1), and towards to splitter (3), which it has a percentage of permeability up to $15 \%$, to incident on mirror (4), then is reflected again, to incident again on splitter (3) and Pass through them. Then towards to splitter (1), it is reflected to the bottom, and then it interferes on two convex lenses which have been put near each other. Then opposite each other, so they accumulate beam on them, and then move the image of the beam to the barrier Figure 6 .

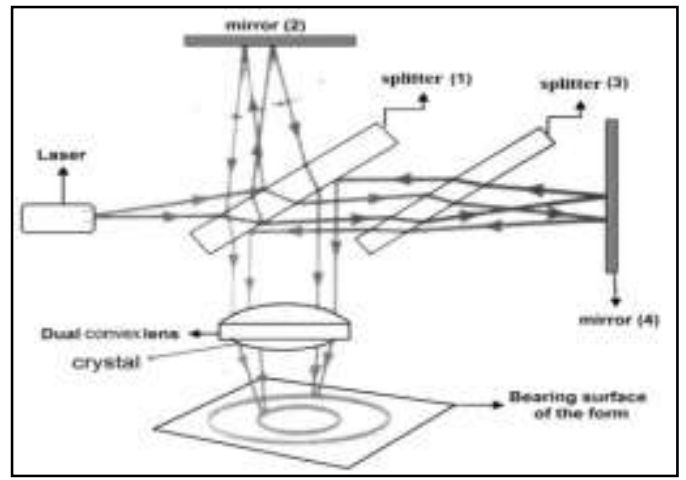

Fig.4: First Optical design, Clear emergence rings as a result of interference ${ }^{[19]}$.

\section{Second Optical Design}

The most important characteristic of the second design figure 7 is to pump two beams of lasers. Through the use two laser devices, which have the same or different types (Superposition) ${ }^{[20-21]}$, where the two devices of laser have been put one above the other, to get out of them two laser beams. then incident on two mirrors $(1,2)$, and each one have been put in front of a beam of laser, at an angle of 45 degrees, in the direction of emanating beams. Therefore the two beam which reflected from mirror (3) are incident on the convex lens, which it move the beam to the crystal, which Working on cracking the beams internally, reflected, and interference with itself, several times in succession, then it come out, to show on the bearing surface.

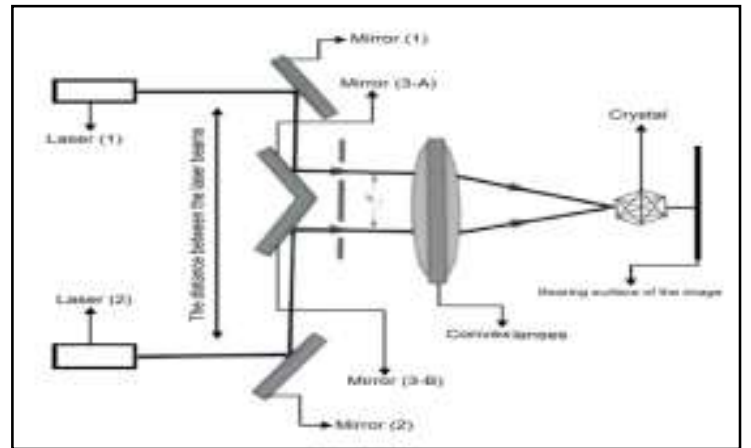

Fig.5: Second Optical Design, Note that the ability of design which collect Mirror 3

with mirror 2.1 on the increase or decrease the distance between the two laser beams ${ }^{[22]}$. 


\section{Third Optical Design}

This type of designs use web cameras or digital cameras to connect with the computer, where the most important characteristic of this registration type, is the digital deal with beam through the presentation of what the camera recorded directly on the computer. In addition to that this design that collect the advantages of the first and second design. The Figure 8 shows the complex optical design, which containing several complex optical systems, where the laser beams appear at the bottom. Stems the beam to the top to incident on slanted mirror at an angle of $45^{\circ}$ degrees, where angle of inclination released this beam forward to pass through the convex lens, then incident on mirror its permeability percentage of $15 \%$, which works as splitter of beam but at the same direction, the original direction of beam -horizontally- is to pass through part of it, and the other part falls back to the lens again, to create a Wavering movement, as for this part which passes-through the mirror, it incident on beam splitter, which it has a permeability percentage $25 \%$. Therefore a part released to the top and the other part is based on the direction of the dumping, which incident on the group of concave and convex lenses to assemble it, then the launch to the Crystal located in front of the lens ${ }^{[23]}$, where the beam is formed inside the crystal through several Splits and reflections, and incident on mirror, which in turn is reflected on the beam once again on two lenses located under the crystal without passing from crystal itself, then moves to the mirror, Then go up to the camera, which has exposure to the beam on computer.

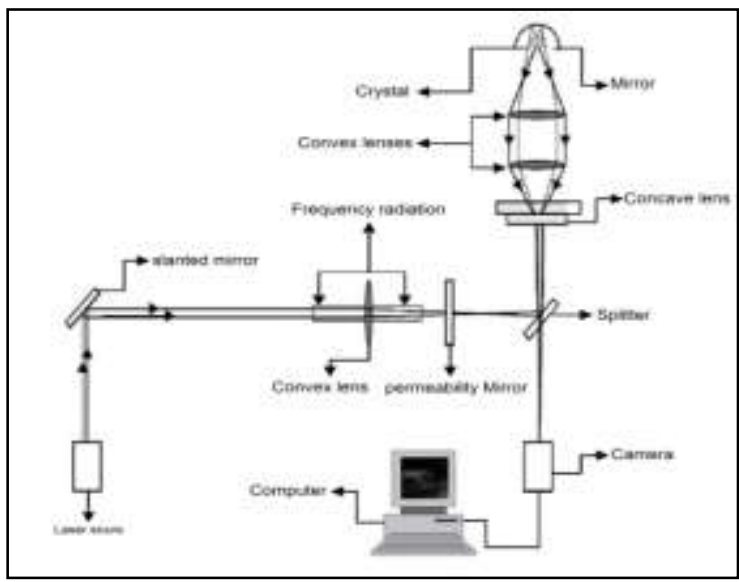

Fig.6: third Optical Design ${ }^{[24]}$

\section{Result and Discussion}

This experiment based on many variables at the level of composition of optical design or at the level of method of exposure to laser beams ${ }^{[25-26]}$. And all variables lead to creating a final visual effect and the visual appearance of the beam has been recorded. The visual effects themselves were influenced when they were visually recorded by registration device, and it is considered a variable in itself ${ }^{[27]}$.

Through this study, we were able to answer the two questions which stated before, through the following results:

\section{First: primary result}

We were able to get the preliminary findings of the three optical designs, as follows:

Figure 9 shows the preliminary result of the first optical design, before control in the variables of the ruling in the configuration, where the figure shows the composition of an arc of laser, which is varied in its degrees, and evident of the arc that the transfers multiplicity of beam, and hitting it with a lot of the reflectors has led to the formation of the distinctive figure.

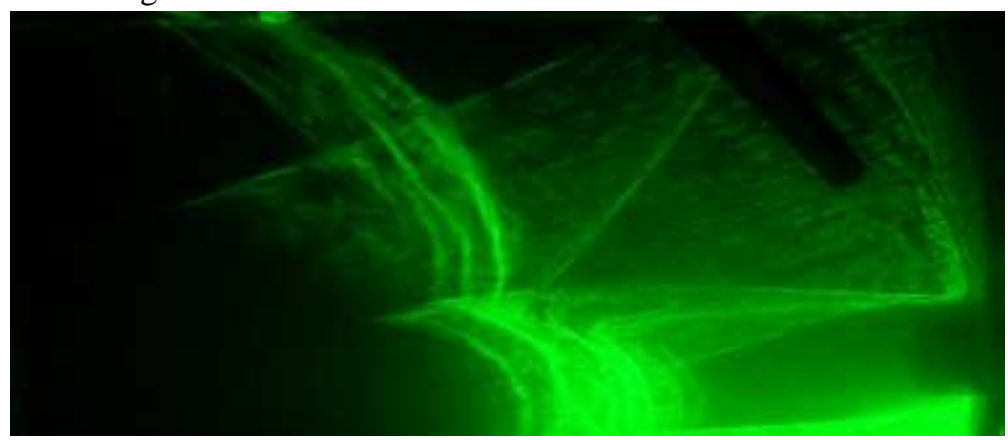

Fig. 7: shows the preliminary result of the first optical design, Shows the waves of Laser light as a result of the much interference, which is going through the radiation ${ }^{[27]}$ 
Figure 10 shows the effect of changing the angles of reflectors, in firsr optical design on change of the final shape of the radiation; it also shows the successive changes resulting from the change optical design. We were able to get the variables from the second optical design, through the crystal, which has worked to break the beams system, and change orientation position of crystal which changed the angle of beam, altered the final shape completely, and made more visual design.

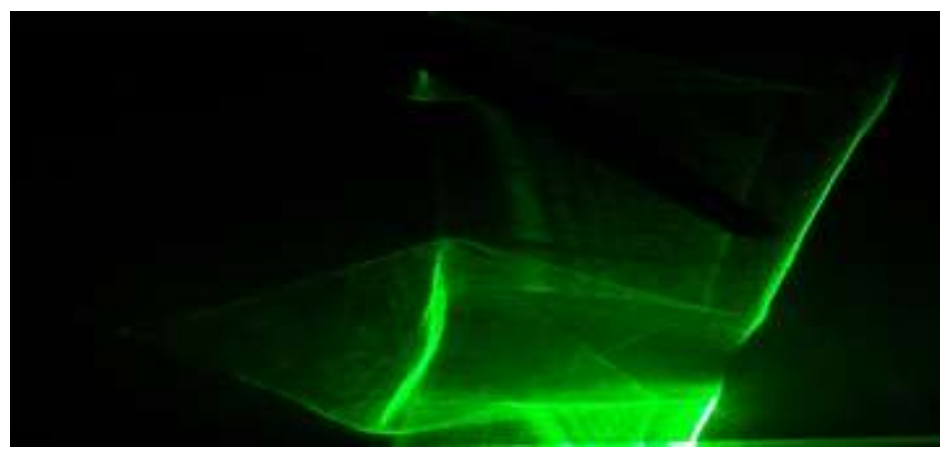

Fig.8: shows the other preliminary result of the first optical design, Clear from the figure the divisions, which the radiation Exposed it, and shows the Interior divisions of, and the researcher was able to exploit, to create a contemporary art form ${ }^{[27]}$.

Figure 11 shows the impact of visual system on the laser beam, where the author took advantage of a double standard optical design to illustrate the difference between the result of this design and previous design. Either in the Third design, the direction changing of the beam splitter is one of the most important variables which could lead to clear different results which they different from the original shape of the optical system. To the extent that they reach both sides of the divided beams into parallel, which results in the possibility to integrate both sides of the beam divided again, leading to impressive results in the control process in sectors of laser radiation, and change the distance between crystal and the laser beam or lens - decrease / increase - helps to produce the -long / short- the time period, which helps in controlling the length of radiation on the visual display.

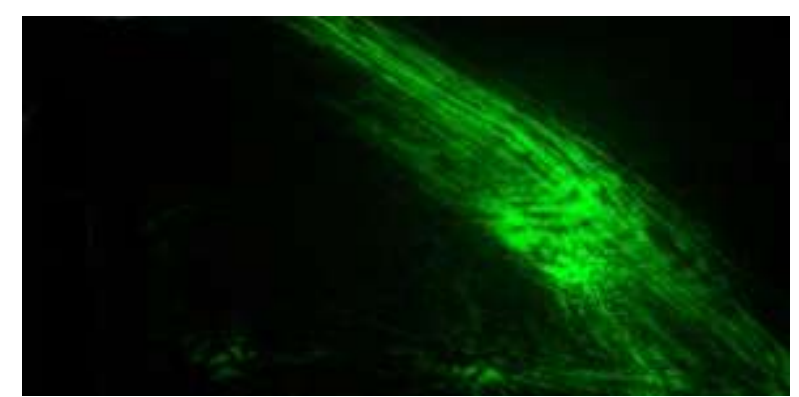

Fig. 9: shows the preliminary result of the second optical design, It is clear fromthe figure above, that it tend to configure to Spaces, unlike the first opticalsystem, in addition to the richness of texture resulting from the operations ofrefraction and dispersion, which beam passed out, Through crystal, and theemergence of the variables on the optical design, has led to a change of basic form ${ }^{[27]}$.

\section{Second: Final Results}

At this stage, we present the findings of the final results, where the use of innovative techniques formerly - for the production of visual art, as a visual Language, and has many concepts and diversified content. Every attempt is originally an artistic experiment of the author started as an attempt depending on the understanding of geometric and wave optics laws and finished as a work of art. We were able to through these attempts reformulate process of the many possibilities of the laser beam, within the artwork. At the same time they serve as a test for the techniques and methods of dealing with the laser beam, to determine the extent of benefit from such techniques to produce form of the visual design.

\section{First attempt}

Figure 14 The first attempt, the name of work : "an entity of light", the number of parts of work : one part, the true dimension of the work : $100 \mathrm{~cm} \times 75 \mathrm{~cm}$, the dimensions of the work after the registration : 100 $\mathrm{cm} \times 50 \mathrm{~cm}$, the severity of work : a laser $(\mathrm{Nd}-\mathrm{YAG})$, implementation technique : the formation of light in space (first optical design), visual type of registration : direct Registration by digital camera, put the tape 
recorder : perpendicular to the visual display area, lighting sensitivity (ISO: 0), the degree of purity of the recorded image: (Resolution: 300 Pixels / Inch), recording time: 7 seconds, and capacity of spatial change: change the status of work change the optical system, the amount of depth in the place: His work on the barrier away from the scene visual distance of $55 \mathrm{~cm}$.

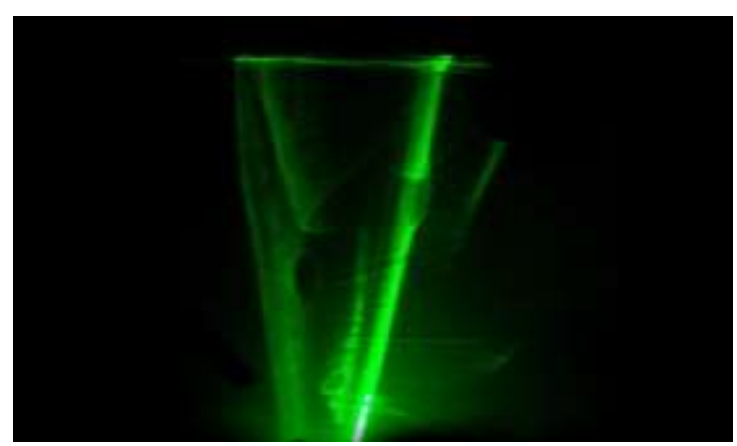

Fig.10: The first attempt, "an entity of light", national institute of laser Enhanced Science, Cairo, Egypt, may $2011^{[27]}$.

Second attempt:

Figure 15 The second attempt, the name of work : "composition", the number of parts of work : one part, the true dimension of the work : $100 \mathrm{~cm} \times 75 \mathrm{~cm}$, the dimensions of the work after the registration : 100 $\mathrm{cm} \times 50 \mathrm{~cm}$, the severity of work : a laser $(\mathrm{Nd}: \mathrm{YAG})$, implementation technique : the formation of light in space (second optical design), visual type of registration : direct Registration by digital camera, put the tape recorder : perpendicular to the visual display area, lighting sensitivity (ISO: 80 ), the degree of purity of the recorded image: (Resolution: 300 Pixels / Inch), recording time: 7 seconds, and capacity of spatial change: change the status of work change the optical system, the amount of depth in the place: His work on the barrier away from the scene visual distance of $75 \mathrm{~cm}$.

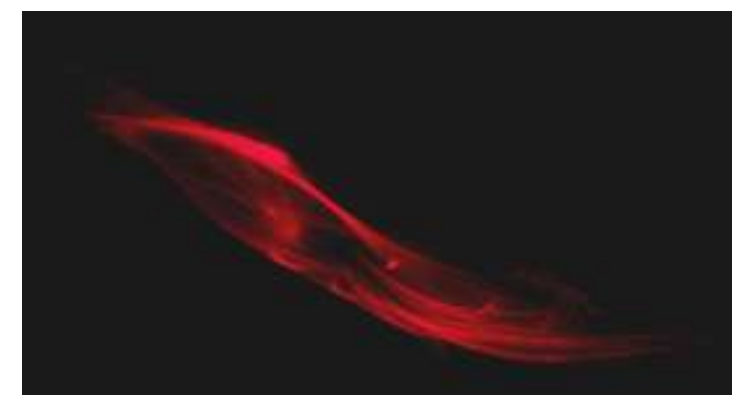

Fig.11: The second attempt, "Composition", national institute of laser Enhanced science, Cairo, Egypt, July $2011^{[27]}$.

\section{Third attempt}

Figure 16 The Third attempt, the name of work : electromagnetic Construction, the number of parts of work : one part, the true dimension of the work : $100 \mathrm{~cm} \times 75 \mathrm{~cm}$, the dimensions of the work after the registration : $100 \mathrm{~cm} \times 50 \mathrm{~cm}$, the severity of work : a laser (Nd-YAG), implementation technique : the formation of light in vacuum (third optical design), visual type of registration : direct Registration by digital camera, put the tape recorder : perpendicular to the visual display area, lighting sensitivity (ISO: 80), the degree of purity of the recorded image: (Resolution: 420 Pixels / Inch), recording time: 7 seconds, and capacity of spatial change: change the status of work change the optical system, the amount of depth in the place: His work on the barrier away from the scene visual distance of $80 \mathrm{~cm}$.

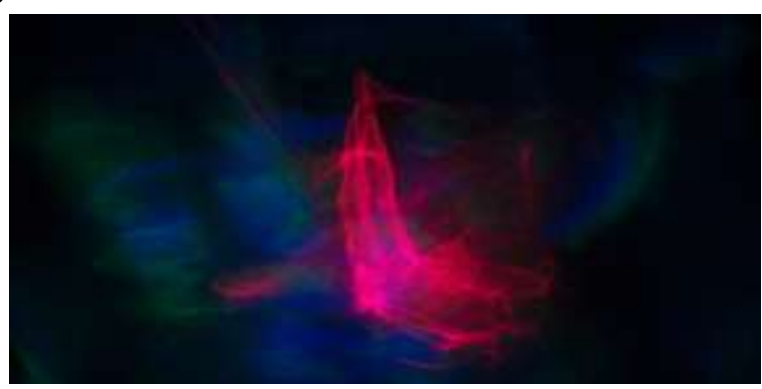

Fig.12: The third attempt, "Electromagnetic construction", national institute of laser enhanced science, Cairo, Egypt, July $2011^{[27]}$. 


\section{Conclusion}

Technology plays an important role in our lives, cannot be dispensed with, and cannot be viewed it as extraneous in our days. When we looked for an answer to two questions which presents in the paper Start, we can say we have found satisfactory answers in the above-mentioned results. Where it is clear from the final artworks in this paper, that the technology is able to enter deeply even in the more things humanitarian(Art), influence it, and vulnerability by it, hence produces a works of art intensive in its concept, distinctive in its shape, new of its kind, and interacts with the time and place.

Therefor we have proved the role of art in utilizing optical science and technology, rather than colliding with them, to present nature in a new way that is more abstract and condense in producing works of art that use technological tools (like laser beams, lenses, beam splitters, ...etc) and control them through the theories and laws of optics. This, from our perspective, is the best example of combining nature and technology based on their mutual background that shows the harmony between science and technology with nature.

But we must be able to note that in this research we were dealing with the final works result as a visual language, and with the elements of these works as a visual vocabulary, not just this, but this works have been built where its elements can be moving at any time, and thus its meaning also can be changing according to the relationship between its visual vocabularies, which control in it the optics laws.

\section{Nomenclature}

Visual art - laser art - design optics

\section{Reference}

[1] NASA, Optics: Light, Color, and Their Uses- an Educator's Guide with Activities in Science and Mathematics Marshall Space Flight Center-2000, P40:44

[2] Shaker Abdul-Hamid, the Strangeness in art, the Egyptian General Book Organization, first edition, Cairo, Egypt, 2011, P 133:134.

[3] Benjamin crowell, Optics, light and matter Fullerton, California, edition, 2.1 Rev, 2004, P 22:25.

[4] [4]- Raifah Mkabbant, conservation collaboration between art and Science, pace university, department of chemistry and physical science, New York, USA, 2000, P 54.

[5] David präkel, photography lighting, Watson-Guptill Publications, USA, 2010, P55-57.

[6] Jonathan benthall, Science and technology in art today, Thames publishing, USA, 2005, P 47.

[7] Mark csele, fundamentals of light source and laser, published by John Wiley \& Sons Inc. Hoboken, New Jersey, USA, 2005 , P 65.

[8] Grouc.D, laser and material, published by John Wiley \& Sons, Inc, Hoboken, New Jersey, USA, 2005, P 100.

[9] Harvey's, Optics, tools and material, published by John Wiley \& Sons, Inc., Hoboken, New Jersey, USA, 2006, P 54

[10] Ophira phonics, laser beam analysis, user guide, new board Corporation brand, UAS, 2012, P21.

[11] Ophira laser measurement group, spiricon laser beam diagnostics, user Guide for LBA-USB, software analysis, version4.84, technical support, 2008, P 132-133.

[12] Carlos B. Roundy, Current Technology of Laser Beam Profile Measurements, Spiricon, Inc. Logan, 2009 , P 56.

[13] Carlos Roundy, propagation factor quantifies laser beam performance, laser focus world, 1999, P 43.

[14] Sasnett, M.W, Characterization of laser beam propagation, Coherent Mode Master Technical Notes, 1990 , P 67.

[15] A scientific experiment of the researcher, national institute of laser enhanced science, Cairo University, Egypt, 2011, P 56.

[16] Ophira laser measurement group, spiricon laser beam diagnostics, user Guide for LBA-USB, technical support, $2008, \mathrm{P} 87$.

[17] Carlson Roundy, propagation factor quantifies laser beam performance, laser focus world, third edition, 2000, P 58.

[18] Carlos Roundy, propagation factor quantifies laser beam performance, laser focus world, $1999, \mathrm{P} 156$

[19] Carlos Roundy, propagation factor quantifies laser beam performance, laser focus world, 1999, P 341.

[20] Ophira laser measurement group, spiricon laser beam diagnostics, user Guide for LBA-USB, software analysis, technical support, 2009, P 212.

[21] A scientific experiment of the researcher, national institute of laser enhanced science, Cairo University, Egypt, 2011.

[22] Curleon.B.Roundy, technology of laser beam profile, spiricon, INC, third edition, 2000, P 65.

[23] Diaa ahmed, laser techniques as an approach to experiment in contemporary visual, drawing and painting dept., faculty of art education, Helwan University, Cairo, Egypt, 2011, P 43.

[24] Migliore L. (Ed.), Laser Material processing, Marcel Dekker Inc, New York, 1996, P 56

[25] Designed by researcher, national institute of laser enhanced science, Cairo University, Egypt, January, 2012, P 87.

[26] Niemz M. H., Laser-tissue interactions, fundamentals and applications, Springer-Verlag, Berlin, 1996, P 98.

[27] Diaa ahmed, National institute of laser enhanced science, Cairo University, Egypt, January, 2012, Thesis, www.niles.edu.eg, www.helwan.edu.eg 